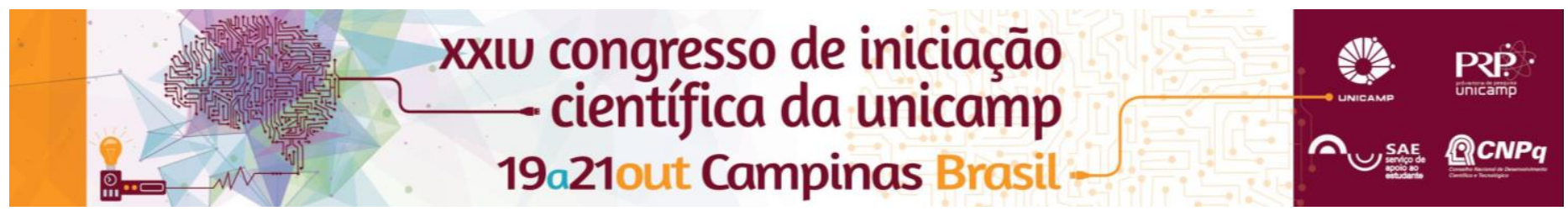

\title{
Autophagy and Metabolic Signaling in Human Skeletal Muscle after Acute Low-Intensity Exercise Cycling with Blood Flow Restriction.
}

\author{
Guilherme D. Telles*, Willian J. Smiles, Miguel S. Conceição, John A. Hawley, Donny M. Camera, Claudia R. \\ Cavaglieri, Mara Patrícia T. Chacon-Mikahil.
}

\begin{abstract}
Autophagy is an intracellular degradative system sensitive to hypoxia and exercise-induced perturbations to cellular bioenergetics. However, the effects of low-intensity endurance-based exercise performed with blood flow restriction (BFR) on cell signaling adaptive responses regulating autophagy and substrate metabolism in human skeletal muscle are unknown. In a randomized cross-over design, 9 young, healthy but physically inactive males completed three experimental trials consisting of either a resistance exercise bout (REX: $4 \times 10$ leg press repetitions, 70\% 1-RM), endurance exercise (END: 30 min cycling, 70\% VO2peak), or low-intensity cycling with BFR (15 min, 40\% VO2peak). A resting muscle biopsy was obtained from the vastus lateralis two weeks prior to the first exercise trial and $3 \mathrm{~h}$ after each exercise bout. END increased ULK1Ser757 phosphorylation above rest and BFR $(\sim 37-51 \%, \mathrm{P}<0.05)$. Following REX there were significant elevations compared to rest ( 348\%) and BFR ( 973\%) for p38 MAPKThr180/Tyr182 phosphorylation $(\mathrm{P}<0.05)$. Parkin content was lower following BFR-cycling compared to REX $(\sim 20 \%, P<0.05)$. There were no exercise-induced changes in select markers of autophagy following BFR. Genes implicated in substrate metabolism (HK2 and PDK4) were increased above rest ( 143-338\%) and BFR-cycling ( 212- 517\%) with END $(P<0.001)$. We conclude that a single bout of low-intensity cycling with BFR is insufficient to induce intracellular "stress" responses (e.g., high rates of substrate turnover, local hypoxia) necessary to activate skeletal muscle autophagy signaling.
\end{abstract}

\section{Key words:}

Blood Flow Restriction, aerobic exercise, autophagy.

\section{Introduction}

Exercise can be broadly categorized as either resistanceor endurance-based contractile activity. Resistance exercise (REX) induces mechanical overload of skeletal muscle to increase myofibre cross-sectional area (hypertrophy) and strength, whereas endurance exercise (END) leads to improvements in whole-body aerobic capacity and increases in peak oxygen uptake ( $\left.\mathrm{VO}_{2 p e a k}\right)$ and skeletal muscle mitochondrial volume-density. An alternative form of exercise stimuli, termed low-intensity END training (i.e., $<50 \%$ of $\mathrm{VO}_{2 \text { peak }}$ ) with blood flow restriction (BFR), can increases both skeletal muscle hypertrophy and $\mathrm{VO}_{2}$ peak. However, little is known about the cellular mechanisms underpinning adaptive responses to endurance-based exercise with BFR. Autophagy, a stress-responsive degradative and "recycling" system is required for the preservation of skeletal muscle mass and mitochondrial biogenesis. END can activate various markers of autophagy in human skeletal muscle, although the combined effect(s) of END with BFR on autophagy activation is unknown. Therefore, the aim of this study was to investigate autophagic cell signalling responses to low-intensity cycling undertaken with BFR and compare these responses to traditional bouts of REX and END.

\section{Results and Discussion}

ULK1 ${ }^{\text {Ser757 }}$ and p38 MAPK Thr180/Tyr182 phosphorylation results are presented in Figure 1. Parkin content was lower following BFR-cycling compared to REX ( 20\%, $\mathrm{P}<0.05)$. There were no exercise-induced changes in select markers of autophagy following BFR. Genes implicated in substrate metabolism (HK2 and PDK4) were increased above rest $(\sim 143-338 \%)$ and BFR-cycling ( 212- 517\%) with END ( $\mathrm{P}<0.001)$.
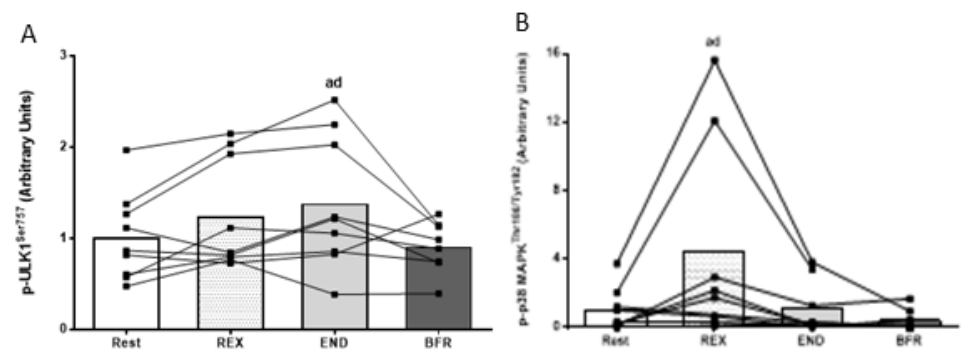

Figure 1. Phosphorylation (p) of ULK1Ser757 (A) and p38 MAPKThr180/Tyr182 (B) $3 \mathrm{~h}$ following a single bout of resistanoe exercise (REX). Endurance exercise (END) or low-intensity oyding with blood-flow restriction (BFR). Values are normalized are presented as individual responses with group mean. Significantly different $(P<0.05)$ vs (a) rest and (d) BFR.

\section{Conclusions}

In conclusion, we provide novel data demonstrating that a single bout of low-intensity BFR cycling failed to stimulate cell signaling responses implicated in autophagy, mitophagy and/or substrate metabolism compared with traditional REX and END. Accordingly, the cumulative stimuli of repeated BFR-cycling bouts are probably necessary to induce the requisite intracellular homeostatic disruptions that lead to adaptation and improved exercise capacity.

\section{Acknowledgement}

${ }^{*}$ FAPESP-IC 2014/16440-2 and BEPE-IC 2015/06994-3. FAEPEX-UNICAMP, LABFEF. Study associated FISEXFEF-UNICAMP and School of Exercise Science of Australian Catholic University (ACU). 